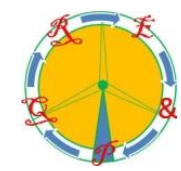

\title{
Multi-Objective Techno-Economic Assessment of Real Life Hydrocarbon Facility Real Power Loss and Power Factor Optimization Using Improved Strength Pareto and Differential Evolutionary Algorithms
}

\author{
M. T. Al-Hajri ${ }^{1}$, M. A. Abido ${ }^{2}$ and M. K. Darwish ${ }^{3}$ \\ ${ }^{1}$ Power Systems, Saudi ARAMCO Oil Company, Dhahran, Kingdom of Saudi Arabia \\ e-mail:muhammad.t.alhajri@gmail.com \\ ${ }^{2}$ Electrical Engineering Department, King Fahad University (KFUPM), Dhahran, Kingdom of Saudi Arabia \\ e-mail:mabido@kfupm.edu.sa \\ ${ }^{3}$ Computer \& Electronic Eng. Department, Brunel University, U.K., Uxbridge, United Kingdom \\ e-mail:mohamed.darwish@brunel.ac.uk
}

\begin{abstract}
In this paper, a techno-economic assessment of a real life hydrocarbon facility electrical system real power loss and grid connection power factor optimization is presented. This optimization was attained by using the Improved Strength Pareto Evolutionary Algorithm (SPEA2) and the Differential Evolutionary Algorithm (DEA). The study is the first of its kind as none of the previous studies were conducted in the context of a real life hydrocarbon facility's electrical system. The hydrocarbon facility's electrical system examined in the study, consists of 275 buses, two gas turbine generators, two steam turbine generators, and large synchronous motors, with both rotational and static loads. For the real life hydrocarbon facility, the performance of the SPEA2 and the DEA were benchmarked in the course of optimizing two competing objectives - power loss and grid connection power factor. The problem was articulated as a constrained nonlinear problem. The constraints were all real values reflecting the system equipment and components' limitations. The results obtained from the research show the efficiency and prospects of the proposed research in solving the described multiple objectives of the study case. Also addressed in this study the annual cost avoidance, due to the study objectives' optimization, based on real fuel value.
\end{abstract}

\section{Keywords}

Improved Strength Pareto Evolutionary Algorithm (SPEA2), differential evolutionary algorithm, power loss optimization, grid connection power factor enhancement, hydrocarbon facility, millions of standard cubical feet of gas (MMscf).

\section{Introduction}

Most of the oil exporting developing countries are facing a challenge associated with the increasing demand for domestic electrical energy. This increase has reached such an alarming level that it mandates action from the governments of the subject countries. An example is illustrated in the Kingdom of
Saudi Arabia, where the average annual increase in electricity demand is $7.4 \%$ [1].

In fact, in these countries, a high percentage of electric generation comes from low efficiency power generation plants, such as the simple cycle steam turbine. This complicates the issue, creating an urgent requirement for the utilization of more efficient plants coupled with a reduction in loss in the transmission and distribution system. In Saudi Arabia, the distribution of plant capacity for electricity generation by technology illustrates that low efficiency simple cycle steam turbine generators make up $32 \%$ of the utility company's generation fleet, while the most efficient combined cycle generators are around $13.8 \%$ of the whole fleet [2].

The aforementioned challenges gave impetus to the idea of studying the potential to use intelligent algorithms in optimizing real hydrocarbon facility power loss and enhancing the grid connection power factor. The study used the real values of the system parameters and practical constraints, which escalated the challenges in finding global solution.

In literature, both the power loss optimization with other contending objectives (multiobjectives) were addressed. In [3], an accelerated particle swarm algorithm was implemented to optimize the multiobjective problem of real power loss reduction, capacitors cost optimization and system stability enhancement. Introducing the weighted sum strategy, the single objective in addressing power system multiobjective was illustrated in [4]. A radial system was incorporated for the verification of the anticipated algorithm in comparison with the other heuristic methods. In [5], evolutionary programming, particle swarm optimization, differential evolution and hybrid differential evolution algorithms were implemented for solving multiobjective active-reactive optimization problems. An IEEE 30-bus and Taiwan Power Company $345 \mathrm{KV}$ simplified system were used to benchmark the performance of the 
selected four evolutionary computation algorithms in solving the problem.

None of the previous studies used a real oil and gas facility power system as the study model. All the previous studies used either IEEE virtual models [5],[6] or utility transmission systems [5],[7] or radial distribution systems [3],[8] as their study models. There are a few studies, which imposed real control variables limitations as constraints such as [8]. The lack of using real system values and limitations, jeopardizes the credibility of the proposed approaches in being applicable in real life.

This paper considers an existing real life hydrocarbon central processing facility electrical power system model for assessing the potential of real power loss (RPL) optimization against the grid connection power factor (GCPF) enhancement- our multiobjective problem. The cost avoidance associated with RPL optimization using real fuel values will be presented in this study. SPEA2 and DEA will be implemented in this study to solve the in-hand multiobjective problem. The performance of the SPEA2 and DEA in addressing the subject problem will be benchmarked. In section 2 of the paper the problem will be formulated as optimization problem with equality and inequality constrains. In section 3, the proposed approach will be presented. In section 4, the study scenarios will be addressed. The study's results analysis and numerical values will be listed in section 5 . In section 6 , the study conclusion and future work will be addressed.

\section{Problem Formulation}

The problem objective is to find the best compromise solution between two competing objectives namely the RPL and the GCPF, while satisfying the system equality and inequality constraints' real life values.

\section{A. Real Power Loss Optimization (RPL)}

The RPL objective is achieved by minimizing $\left(P_{\text {Loss }}\right)$, the real power loss within the transmission and distribution lines of the system. This objective function $\left(J_{l}\right)$ can be expressed in terms of the power flow loss between two buses, $V_{i}$ and $V_{j}$ as follows:

$J_{I}=\operatorname{Minimize}\left(P_{\text {Loss }}\right)=\left(\sum_{k=1}^{n l} g_{k}\left[V_{i}^{2}+V_{j}^{2}-2 V_{i} V_{j} \cos \left(\delta_{i}-\delta_{i}\right)\right]\right)$

where $n l$ is the number of transmission and distribution lines; $g_{k}$ is the conductance of the $k^{\text {th }}$ line, $V_{\mathrm{i}} \angle \delta_{\mathrm{i}}$ and $V_{j} \angle \delta_{\mathrm{j}}$ are the voltage at end buses $i$ and $j$ of the $k^{\text {th }}$ line, respectively [6] [9].

\section{B. Grid Connection Power Factor (GCPF) Enhancement}

The system regulator in a regulated electricity market mandates a penalty on any plant that has GCPF exceeding a certain limit. This is needed to regulate the reactive power absorbed or injected into the grid. The limit is usually set equal to 0.85 lagging or leading. Additional reactive power injected or absorbed will be subject to a penalty tariff. In this study, the GCPF will be pushed to unity $[1.0 \mathrm{PF}=\cos (\theta)]$ and accordingly the PF angle $|\theta|$ will be zero (0). Therefore, the GCPF angle index can be expressed as follows:

$$
\mathrm{L}_{\mathrm{GCPF}}=|\theta|
$$

Hence, the GCPF objective (J2) is expressed as follows:

$$
J_{2}=\operatorname{Minimize}\left(L_{G C P F}=|\theta|\right)=\operatorname{Maximize}(P F)
$$

\section{Problem Equality and Inequality Constrains}

The system constrains are divided into two categories: equality constrains and inequality constrains [3],[5]-[6]. Details are as follows:

\section{C.1 Equality Constrains}

These constrains represent the power load flow equations. The balance between the active power injected $\mathrm{P}_{\mathrm{Gi}}$, the active power demand $\mathrm{P}_{\mathrm{Di}}$ and the active power loss $\mathrm{P}_{\mathrm{li}}$ at any bus $\mathrm{i}$ is equal to zero. The same balance apply for the reactive power $\mathrm{Q}_{\mathrm{Gi}}, \mathrm{Q}_{\mathrm{Di}}$, and $\mathrm{Q}_{\mathrm{li}}$. These balances are presented as follows:

$$
\begin{aligned}
& \mathrm{P}_{\mathrm{Gi}}-\mathrm{P}_{\mathrm{Di}}-\mathrm{P}_{\mathrm{li}}=0 \\
& \mathrm{Q}_{\mathrm{Gi}}-\mathrm{Q}_{\mathrm{Di}}-\mathrm{Q}_{\mathrm{li}}=0
\end{aligned}
$$

The above equations can be detailed as follow:

$$
\begin{aligned}
& \mathrm{P}_{\mathrm{Gi}}-\mathrm{P}_{\mathrm{Di}}-V_{i} \sum_{j=\mathbf{1}}^{N B} V_{j}\left[G_{i j} \cos \left(\delta_{i}-\delta_{j}\right)+B_{i j} \sin \left(\delta_{i}-\delta_{j}\right)\right]=0 \\
& \mathrm{Q}_{\mathrm{Gi}}-\mathrm{Q}_{\mathrm{Di}}-V_{i} \sum_{j=\mathbf{1}}^{N B} \boldsymbol{V}_{\boldsymbol{j}}\left[G_{i j} \sin \left(\delta_{i}-\delta_{j}\right)-B_{i j} \cos \left(\delta_{i}-\delta_{j}\right)\right]=0
\end{aligned}
$$

where $i=1,2, \ldots, \mathrm{NB} ; \mathrm{NB}$ is the number of buses; $\mathrm{P}_{\mathrm{G}}$ and $\mathrm{Q}_{\mathrm{G}}$ are the generator real and reactive power, respectively; $\mathrm{P}_{\mathrm{D}}$ and $\mathrm{Q}_{\mathrm{D}}$ are the load real and reactive power, respectively; $G_{i j}$ and $B_{i j}$ are the conductance and susceptance between bus $i$ and bus $j$, respectively.

\section{C.2 Inequality Constrains}

These constrains represent the system operating constrains posted in Table I and they are as follow:

a. Generator and synchronous motor voltages; $\mathrm{V}_{\mathrm{G}}$ and $\mathrm{V}_{\text {Synch }}$; their reactive power outputs; $\mathrm{Q}_{\mathrm{G}}$ and $\mathrm{Q}_{\text {Synch }}$.

b. The transformers taps.

c. The load buses voltages $\mathrm{V}_{\mathrm{L}}$. 


\section{Multiobjective Formulation}

Each day there are many situations in which there are numerous objectives to be met. These objectives compete against each other. The solution for one objective may not provide a solution for another objective. The nature of the objectives calls for the development of numerous optimal solutions. The reason for this is that no single optimal solution can be regarded as superior over any other concerning every part of the objective's functions. These type of solutions are named as Pareto-optimal solutions. A multiobjective optimization problem subject to constraints is termed as follows:

$$
\begin{aligned}
& \text { Minimize } f_{i}(x) \quad i=1, \ldots \ldots, N_{o b j} \\
& \text { Subject to: } \begin{cases}g_{j}(x)=0 & j=1, \ldots \ldots, M \\
h_{k}(x) \leq 0 & k=1, \ldots \ldots, K\end{cases}
\end{aligned}
$$

where $f_{i}$ is the objective functions of $\mathrm{i}^{\text {th }}$ objective function, $\mathrm{x}$ denotes the decision vector representing a solution, $\mathrm{N}_{\text {obj }}$ represents the objective numbers, $M$ represents the equality constraints and $\mathrm{K}$ represents the inequality constraints. In any optimization problem, which involves multi-tasking, any two solutions such as $\mathrm{x}^{1}$ and $\mathrm{x}^{2}$ can have only two possibilities in terms of their mathematical relationship to each other. Either one can dominate the other, or none of them dominates each other. In the problem of minimization, deprived of the loss of generality, and under these conditions, a solution $\mathrm{x}^{1}$ dominates $\mathrm{x}^{2}$, if

$$
\begin{aligned}
& \text { 1. } \forall i \in\left\{1,2, \ldots, N_{o b j}\right\}: f_{i}\left(x^{1}\right) \leq f_{i}\left(x^{2}\right) \\
& \text { 2. } \exists j \in\left\{1,2, \ldots, N_{o b j}\right\}: f_{j}\left(x^{1}\right)<f_{j}\left(x^{2}\right)
\end{aligned}
$$

Violation of any above-mentioned conditions, solution $\mathrm{x}^{1}$ does not dominate the solution $\mathrm{x}^{2}$. If $\mathrm{x}^{1}$ dominates solution $\mathrm{x}^{2}$ then, $\mathrm{x}^{1}$, represents the non-dominated solution. Thus, Paretooptimal is the non-dominated solution over all other solutions and constructs the set of Pareto-optimal or front Paretooptimal. Considering all the above objectives and constraints, the study problem may be precisely devised as a non-linear constrained optimization problem described as per the following equations:

Optimize (minimize) $J^{l}$ and $J^{2}$

$$
\text { Subject to : }\left\{\begin{array}{c}
g(x, u)=0 \\
|h(x, u)| \leq 0
\end{array}\right.
$$

Where:

$\mathrm{x}$ : is the vector of dependent variables consisting of load bus voltage $\mathrm{V}_{\mathrm{Li}}$, generator reactive power outputs $\mathrm{Q}_{\mathrm{Gi}}$ and the Synchronous motors reactive Power $\mathrm{Q}_{\text {synchi. }}$ As a result, $\mathrm{x}$ can be expressed as

$$
\mathrm{x}^{\mathrm{T}}=\left[\mathrm{V}_{\mathrm{L} 1} . . \mathrm{V}_{\mathrm{LNL}}, \mathrm{Q}_{\mathrm{Gi}} \ldots \mathrm{Q}_{\mathrm{GNG}}, \mathrm{Q}_{\text {Synchi } \left.\ldots . Q_{\text {SynchNSynch }}\right]}\right]
$$

$\mathrm{u}$ : is the vector of control variables consisting of generator voltages $\mathrm{V}_{\mathrm{G}}$, transformer tap settings $\mathrm{T}$, and synchronous motors voltage $\mathrm{V}_{\text {Synch. }}$ As a result, $\mathrm{u}$ can be expressed as

$$
\mathrm{u}^{\mathrm{T}}=\left[\mathrm{V}_{\mathrm{G} 1} . . \mathrm{V}_{\mathrm{GNL}}, \mathrm{T}_{1} \ldots \mathrm{T}_{\mathrm{NT}}, \mathrm{V}_{\text {Synch } 1 . .} \mathrm{V}_{\text {SynchNL }}\right]
$$

$\mathrm{g}$ : are the equality constrains.

h: are the inequality constrains.

Table I - System Inequality Constrains

\begin{tabular}{|l|c|c|}
\hline \multicolumn{1}{|c|}{ Description } & Lower Limit & Upper Limit \\
\hline GTG Terminal Voltage $\left(\mathrm{V}_{\mathrm{GTG}}\right)$ & $90 \%$ & $105 \%$ \\
\hline STG Terminal Voltage $\left(\mathrm{V}_{\mathrm{STG}}\right)$ & $90 \%$ & $105 \%$ \\
\hline GTG Reactive Power $\left(\mathrm{Q}_{\mathrm{GTG}}\right)$ Limit & -62.123 & $95.72 \mathrm{MVAR}$ \\
& MVAR & \\
\hline STG-1 Reactive Power $\left(\mathrm{Q}_{\mathrm{STG}}\right)$ Limit & $-22.4 \mathrm{MVAR}$ & $20.92 \mathrm{MVAR}$ \\
\hline STG-2 Reactive Power $\left(\mathrm{Q}_{\mathrm{sTG}}\right)$ Limit & $-41.9 \mathrm{MVAR}$ & $53.837 \mathrm{MVAR}$ \\
\hline Captive Synch. Motors Terminal Voltage & $90 \%$ & $105 \%$ \\
\hline Synch. Motors Terminal Voltage $\left(\mathrm{V}_{\mathrm{Sychn}}\right)$ & $90 \%$ & $105 \%$ \\
\hline Causeway downstream Buses Voltage & $95 \%$ & $105 \%$ \\
\hline All Load Buses Voltage & $90 \%$ & $105 \%$ \\
\hline Main Transformer Taps & $+16(+10 \%)$ & $-16(-10 \%)$ \\
\hline Generators Step-Up Transformer Taps & $+8(+10 \%)$ & $-8(-10 \%)$ \\
\hline
\end{tabular}

\section{The Proposed Approach}

\section{A. Improved Strength Pareto Evolutionary Algorithm (SPEA2)}

The following steps describe the SPEA2 evolutionary process [6].

Step 1: Initialization

The population $\mathrm{P}_{0}$ is generated with $K$ size and a vacant annals (external) Pareto-optimal set $\overline{P_{0}}$ with $\bar{K}$ size.

Step 2: Updating of external pareto set In order to bring an updated set of the external Pareto ptimal set, the following steps are to be followed:

(a) The population of non-dominated individuals are highlighted and reproduced to the external Pareto set.

(b) Look for the set of external Pareto, designed for the nondominated individuals.

(c) If condition $\left(\overline{P_{t+1}}\right)<\bar{K}$ is satisfied, keep the individuals with higher fitness values until $\left|\overline{P_{t+1}}\right|=\bar{K}$ is satisfied.

(d) If $\left(\overline{P_{t+1}}\right)>\bar{K}$, truncation procedure is needed to remove individuals from $\left(\overline{P_{t+1}}\right)$ in anticipation of $\left|\overline{P_{t+1}}\right|=\bar{K}$.

Step 3: Assignment of fitness

The fitness values of the individuals are calculated in both the external Pareto set $\overline{\mathrm{P}}_{\mathrm{t}}$ and the population $P_{t}$ as follows:

(a) $S t(i)$; strength value, is assigned to all individuals $i$ inside the external pareto set $\overline{\mathrm{P}}_{\mathrm{t}}$ and the population $\mathrm{P}_{\mathrm{t}}$. St $(i)$ signifies the unit, which $i$ dominates and is expressed as follows: 


$$
\mathrm{S} t(i)=\mid\left\{j, j \in P_{t}+\overline{P_{t}}, \wedge i>j\right\}
$$

Then, the raw fitness $\mathrm{R}_{\mathrm{w}}(i)$ with respect to an individual $i$ can be measured as follows:

$$
\mathrm{R}_{w}(i)=\sum_{j \in P_{t}+\bar{P}_{t, j>i}} S t(j)
$$

The raw fitness of an individual is obtained with respect to the strength of its dominators in the archive and population.

(b) The distances between an individual $i$ and the entire $j$ individuals, in the course of external and population sets, are listed. Then, the list is sorted in a cumulative manner, the distance to the $\mathrm{m}^{\text {th }}$ individual, consequently $m=\sqrt{\bar{K}+K}$ is represented as $\sigma_{i}^{m}$. Then, $\mathrm{D}(i)$ (density) is calculated for each $i$ :

$$
\mathrm{D}(i)=\frac{1}{\sigma_{i}^{m}+2}
$$

The addition of integer 2 is made in the denominator to certify that the value of $\mathrm{D}(i)$ is larger than zero and is $<1$. The fitness value $i$ of an individual is expressed as follows:

$$
\mathrm{F}(i)=\mathrm{R}_{\mathrm{w}}(i)+\mathrm{D}(i)
$$

\section{Step 4: Selection}

Two individuals have been selected on a random basis from the restructured external set $\overline{\mathrm{P}_{\mathrm{t}+1}}$. In light of their fitness values, the best one is selected and is copied to the mating pool.

Step 5: Crossover and Mutation

The simple crossover operation and random mutation operation is performed on the basis of the probabilities for the generation of the novel population. The offspring $\mathrm{H}=\left(\mathrm{h}_{1}, \ldots . \mathrm{h}_{\mathrm{i}}, \ldots . \mathrm{h}_{\mathrm{n}}\right)$ is generated in the simple crossover by establishing a vertical crossover position for two parents' chromosomes. Then, the two new chromosomes are built. In the random mutation method the new gene is generated randomly from the genes' domain.

Step 6: Looping back

Stopping criteria is checked. If not satisfied, then copy the new offspring population to the old one.

Step 7: Termination

The criteria for termination is checked. If satisfied, apply the fuzzy set theory to identify the best possible compromise solution out of the pareto external set. Figure 1 illustrates SPEA2 evolutionary steps.

\section{B. Differential Evolutionary Algorithm (DEA)}

DEA utilizes special differential operators in creating the offspring individuals from the parent individuals' population in place of the classical crossover and mutation operators used in the SPEA2. In DEA there are two control parameters, which are the mutation constant $\mathrm{F}$ and the crossover constant $\mathrm{C}$. Also in DEA, the mutations are performed before crossover. DEA's first three evolutionary process steps are similar to the SPEA2 ones [9], [10]. The remaining steps are as described below:

\section{Step 4: Mutation}

Different from the SPEA2, in which the individuals to be subjected to crossover and mutation are selected from the front pareto optimal set, in DEA the individuals are selected from the population. In DEA, mutations are performed using the $\mathrm{DE} / \mathrm{rand} / 1$ mutation technique. $\mathrm{V}_{\mathrm{i}}(t)$, the mutated vector, is created for each population member $\mathrm{X}_{\mathrm{i}}(t)$ set by randomly selecting three individuals $\mathrm{x}_{\mathrm{r} 1}, \mathrm{x}_{\mathrm{r} 2}$ and $\mathrm{x}_{\mathrm{r} 3}$ and not corresponding to the current individual $\mathrm{x}_{\mathrm{i}}$. Then, a scalar number $\mathrm{F}$ is used to scale the difference between any two of the selected individuals. The resultant difference is added to the third selected individual. The mutation process can be written as:

$$
\mathrm{V}_{i, j}(t)=\mathrm{x}_{r l, j}(t)+F \cdot\left[\mathrm{x}_{r 2, j}(t)-\mathrm{x}_{r 3, j}(t)\right]
$$

The value of $F$ is usually selected between 0.4 and 1.0. In this study F was set to be $0.5(50 \%)$.

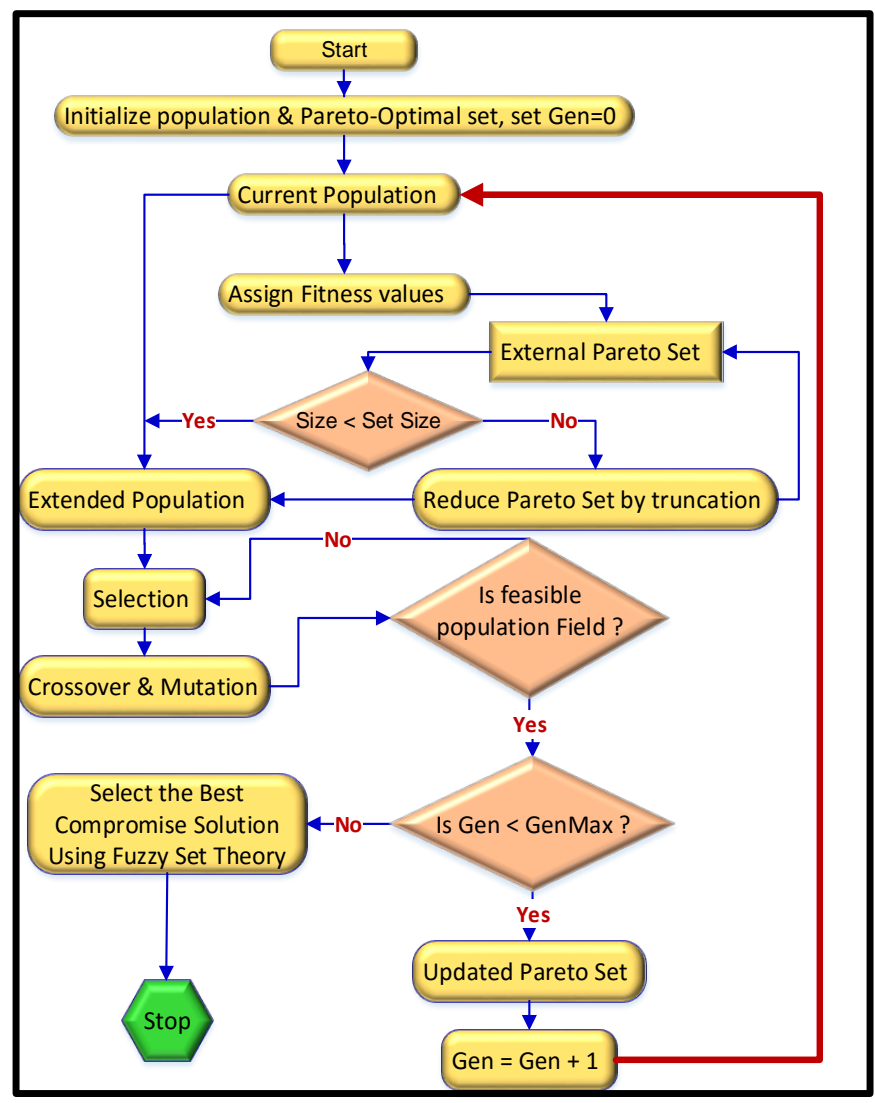

Fig. 1. SPEA2 evolutionary process flowchart

Step 5: Crossover

Perform the binomial crossover, which can be expressed as follows: 


$$
u_{i, j}(t)=\left\{\begin{array}{lr}
v_{i, j}(t) \text { if rand }(0,1)<C R \\
x_{i, j}(t) & \text { else }
\end{array}\right.
$$

$\mathrm{CR}$ is the crossover control parameter and it is usually set within the range $[0,1]$. The child $\mathrm{u}_{\mathrm{i}, \mathrm{j}}(t)$ will contend with its parent $\mathrm{x}_{\mathrm{i}, \mathrm{j}}(t)$. CR is set equal to $0.9(90 \%)$ in this study.

Step 6: Selection

The procedure for the selection is as follows:

$$
\begin{array}{rll}
\mathrm{x}_{i}(t+1)=\mathrm{u}_{i}(t) \quad \text { condition } & f\left(\mathrm{u}_{i}(t)\right) \leq f\left(\mathrm{x}_{i}(t)\right) \\
\mathrm{x}_{i}(t+1)=\mathrm{x}_{i}(t) & \text { condition } & f\left(\mathrm{x}_{i}(t)\right) \leq f\left(\mathrm{u}_{i}(t)\right)
\end{array}
$$

where $f()$ is the objective function to be minimized. The last two steps are similar to SPEA2 step 6 and step 7. Figure 2 demonstrates DEA evolutionary steps.

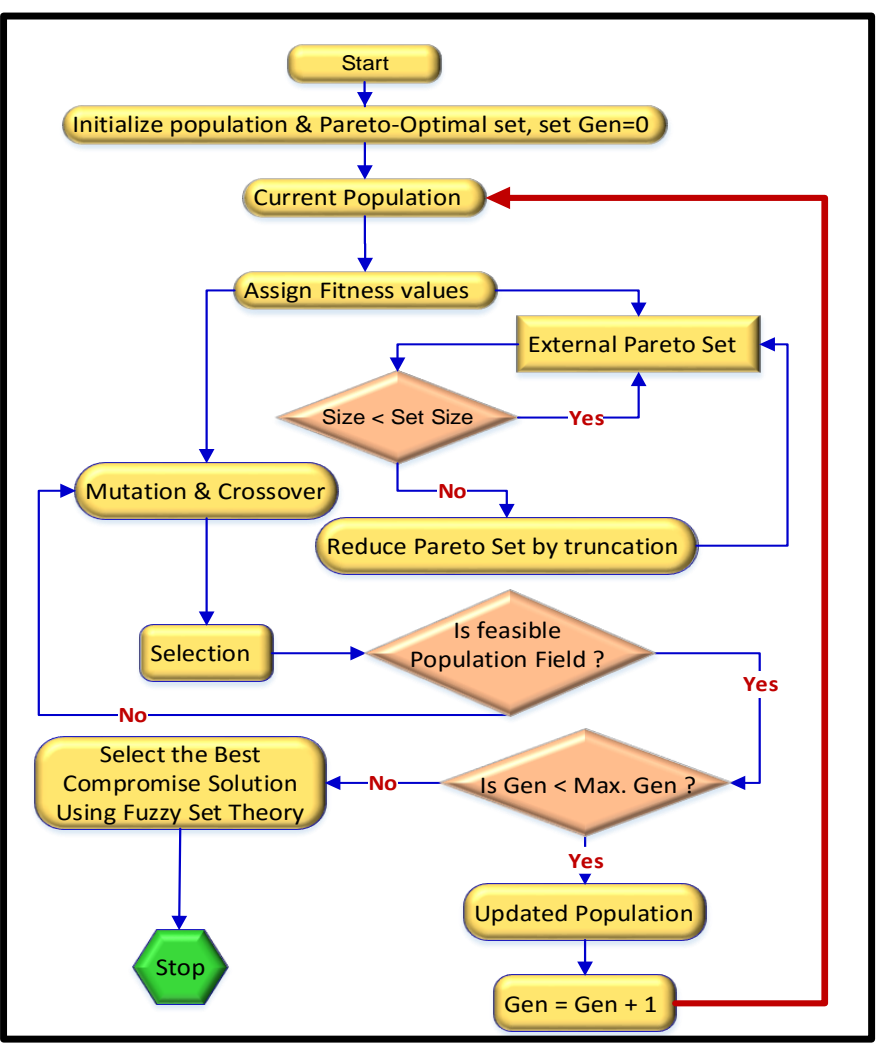

Figure 2. DEA algorithm evolution process flowchart

\section{Front Pareto Set Reduction by Truncation}

In some cases, the front pareto optimal set can be very large. In such cases, reducing the set of nondominated solutions without destroying the characteristics of the trade-off front is necessary. One means to accomplish this goal is the truncation technique. In the truncation technique, a minimum distance based algorithm [6] is utilized to reduce the size of the front pareto set to a manageable size, i.e. the specified external pareto set size. At each iteration an individual $i$ is chosen for removal from the external pareto set $P_{t+1}$.

\section{Front Pareto Set Reduction by Truncation}

The decision makers need a trusted selection technique to identify the best compromise solution out of the pareto-optimal set of non-dominated individuals [6] and [9]. The fuzzy set theory will help in presenting one solution to the decision makers as the best compromise solution. Due to the decision makers' inexact conclusion, the $i^{\text {th }}$ objective function $J_{i}$ is represented by a membership function $\mu_{i}$ defined as in equation

$$
\mu_{i}= \begin{cases}1 & J_{i} \leq J_{i}^{\min } \\ \frac{J_{i}^{\max }-J_{i}}{J_{i}^{\max }-J_{i}^{\min }} & J_{i}^{\min }<J_{i}<J_{i}^{\max } \\ 0 & J_{i} \geq J_{i}^{\max }\end{cases}
$$

where $J_{i}^{\min }$ and $J_{i}^{\max }$ are the minimum and maximum value of the $i^{\text {th }}$ objective function among all non-dominated solutions, respectively. For each non-dominated solution $k$, the normalized membership function $\mu^{k}$ is calculated as follows:

$$
\mu^{k}=\frac{\sum_{i=1}^{N_{o b j}} \mu_{i}^{k}}{\sum_{k=1}^{M} \sum_{i=1}^{N_{o b j}} \mu_{i}^{k}}
$$

where $M$ is the number of non-dominated solutions. The best compromise solution is that having the maximum value of $\mu^{k}$.

\section{Study Scenarios}

In this paper, three scenarios were studied: the base case scenario- business as usual (BAU), the optimal case scenario with all generators are online, and the optimal case scenario with two generators are offline. In the optimal cases the best system parameters (chromosomes) that make the best front pareto set are obtained.

\section{A. Base Case Scenario}

The BAU scenario was simulated to be benchmarked with the two optimal scenarios. Following are some of the BAU mode parameters:

1) The utility bus and generators terminal buses were set at unity p.u. voltage.

2) All the synchronous motors were set to operate very close to the unity power factor. 
3) All downstream distribution transformers and the captive synchronous motors transformers; off-load tap changers; were put on the neutral tap.

4) The causeway substations main transformers taps were raised to meet the very conservative voltage constrains at these substations downstream buses; $\geq 0.95$ p.u. Refer to Table II below.

Table II - The Selected Feasible Transformers Taps Value

\begin{tabular}{|l|c|}
\hline \multicolumn{1}{|c|}{ Substation Number } & Transformer Tap \\
\hline Causeway Substation\#1 & $+3(1.019$ p.u. $)$ \\
\hline Causeway Substation\#2 & Neutral $(1.0$ p.u. $)$ \\
\hline Causeway Substation\#3 & $+3(1.019$ p.u. $)$ \\
\hline Main Substation Transformers & $+1(1.006$ p.u. $)$ \\
\hline
\end{tabular}

\section{B. Optimal Case Scenario with All Generators Online}

In this scenario all the generators were assumed to be online. An initial 100 populations of feasible chromosomes (individuals) which meet both the buses voltage and synchronous machine reactive power constrains were identified. The feasible populations with their associated chromosomes were subject to the SPEA2 and DEA evolutionary process of 100 generations guided by the objective functions $J_{1}$ and $J_{2}$. The system parameters and the front pareto set values associated with the best compromised solutions the studied scenarios were identified

\section{Optimal Case Scenario with Two Generators are Offline}

In this scenario two generators (one gas turbine generator and one steam turbine generator) were assumed to be offline. All others parameters are identical to optimal case scenario with all generators are online.

\section{Results Analysis And Discussions}

The results from the three scenarios, BAU case, all generators online and two generators are offline will be analysed in two categories: the system parameters analysis and the economic analysis.

\section{A. System Parameters Analysis}

The hydrocarbon facility simplified electrical system model, which is studied in this paper, is shown in Figure 3. The evolution of the objective functions $\left(J_{l}\right.$ and $\left.J_{2}\right)$ values over the SPEA2 and DEA evolutionary process is captured in Figure 4. In the all generators online scenario the SPEA2 converted to it optimal $J_{l}$ value of $1.918 \mathrm{MW}$ and $J_{2}$ value of $0.359^{\circ}$ while the DEA converted to better $J_{l}$ value of $1.898 \mathrm{MW}$ and $J_{2}$ value of $4.12^{0}$. So, DEA converted to better $J_{l}$ value but SPEA2 converted to better $J_{2}$ value. In the two generators offline scenario DEA converted to again better $J_{l}$ value of $2.943 \mathrm{MW}$ while the SPEA2 converted to it optimal $J_{l}$ value of $2.987 \mathrm{MW}$.
In this scenario, DEA produced better $J_{l}$ value and equal $J_{2}$ value when compared with SPEA2. The evolution process for both the SPEA2 and the DEA were repeated many times to confirm the obtained results.

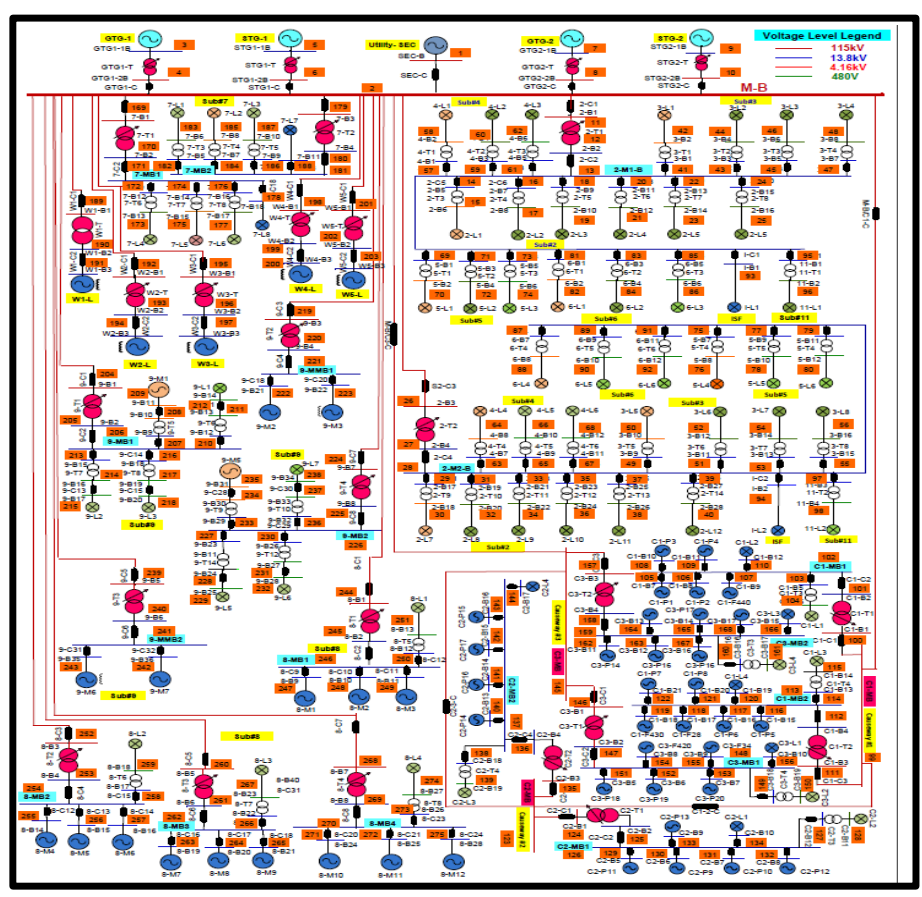

Fig. 3 Simplified electrical system of the hydrocarbon facilty

In Table III, comparison of the objective functions' values are posted for the three studied scenarios.

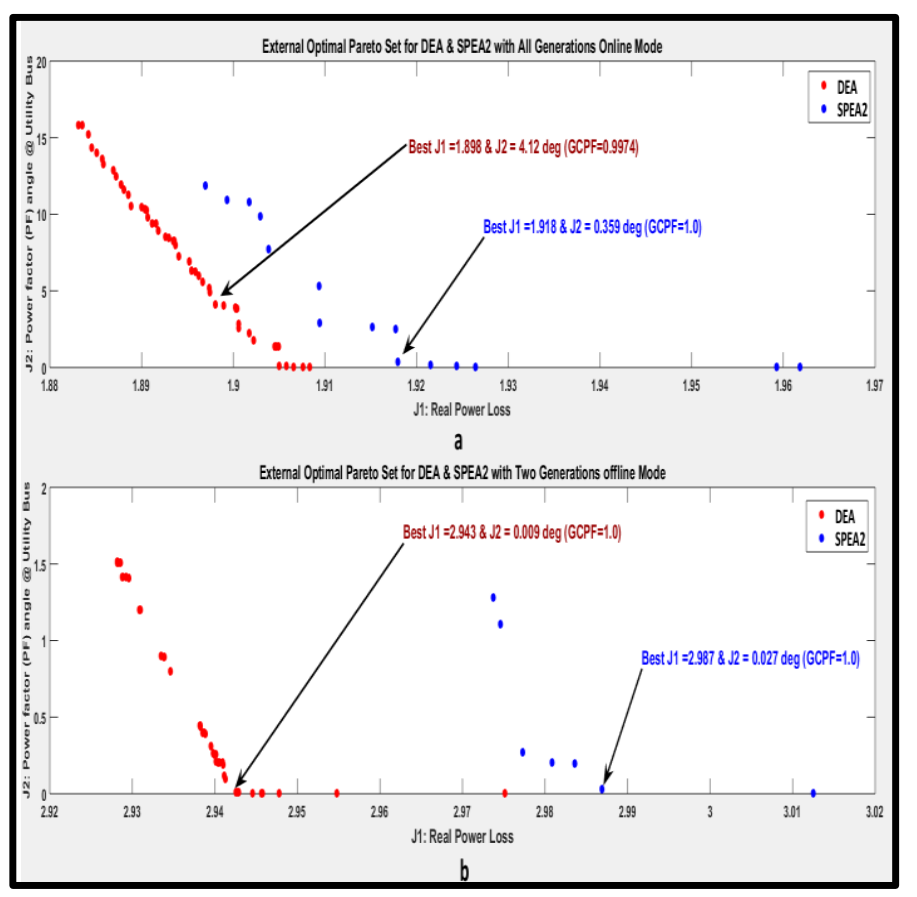

Fig. 4. $\mathrm{J}_{1}$ and $\mathrm{J}_{2}$ front-pareto optimal sets for SPEA2 and DEA 
Table III - The $J_{1}$ and $J_{2}$ Values for the Studied Three Scenarios

\begin{tabular}{|c|c|c|c|}
\hline Generation Mode & Case \# & $J_{l}$ Value & $J_{2}$ Value \\
\hline All online & BAU & 2.134 & 0.7704 \\
\hline Two offline & BAU & 3.219 & 0.9746 \\
\hline All online & SPEA2 & 1.918 & $\mathbf{1 . 0 0}$ \\
\hline All online & DEA & $\mathbf{1 . 8 9 8}$ & 0.9974 \\
\hline Two offline & SPEA2 & 2.987 & 1.00 \\
\hline Two offline & DEA & $\mathbf{2 . 9 4 3}$ & 1.00 \\
\hline
\end{tabular}

\section{B. Economic Analysis}

Table IV summarizes the cost avoidance associated with all three scenarios, giving the two generation modes. The fuel value of $\$ 3.5$ for each one million BTU is used. The calculated costs are the systems' real power loss expenses- the real power injected to (revenue) or absorbed from (expense) the grid, the reactive power injected to or absorbed from the grid penalty expense, if $|\mathrm{PF}|<0.85$ limit and the cost balance of adding these three expenses or revenues. In all generations' online scenario, DEA had a better cost balance over SPEA2's $\$ 11,571,336$, with potential revenue of $\$ 1,010,178$ over the BAU's $\$ 10,551,157$ value. On the other hand, when applying the two generations offline scenario, DEA had less expense cost balance - $\$ 66,847,245$ - compared to SPEA2.

Table IV-Economic Analysis for the Studied Three Scenarios

\begin{tabular}{|c|c|c|c|c|c|}
\hline $\begin{array}{c}\text { Generation } \\
\text { Mode }\end{array}$ & Case\# & $\begin{array}{c}\text { Real } \\
\text { Power } \\
\text { Loss Cost }\end{array}$ & $\begin{array}{c}\text { Real Power } \\
\text { Injected into } \\
\text { the Grid } \\
\text { Cost }\end{array}$ & $\begin{array}{c}\text { Reactive } \\
\text { Power } \\
\text { Injected into } \\
\text { the Grid } \\
\text { Cost }\end{array}$ & Cost Balance \\
\hline All online & BAU & $(635,982)$ & $12,061,076$ & $(873,937)$ & $10,551,157$ \\
\hline Two offline & BAU & $(959,485)$ & $(66,059,417)$ & 0.00 & $(67,018,903)$ \\
\hline All online & SPEA2 & $(571,637)$ & $12,130,636$ & 0.00 & $11,558,999$ \\
\hline All online & DEA & $(565,709)$ & $12,137,045$ & 0.00 & $\mathbf{1 1 , 5 7 1 , 3 3 6}$ \\
\hline Two offline & SPEA2 & $(890,231)$ & $(65,984,534)$ & 0.00 & $(66,874,765)$ \\
\hline Two offline & DEA & $(877,008)$ & $65,970,236)$ & 0.00 & $(\mathbf{6 6 , 8 4 7 , 2 4 5 )}$ \\
\hline
\end{tabular}

\section{Conclusion and Future Work}

In this paper, the objective functions optimization of the proposed multiobjective problem was assessed for two different generation scenarios when applying SPEA2 and DEA algorithms. The real power loss objective was addressed as a multiobjective problem considering the GCPF ehnacment objective. Unique to this study, over previous literature studies, was that a real life hydrocarbon facility's electrical system was utilized. The fuzzy set theory was implemented to extract the best compromise value out of the optimal objective values in the front pareto optimal set. The effects of the generation modes and the applied optimization algorithms in addressing the study objective functions' values were reviewed. Both the technical and economic analysis showed the superiority of DEA over SPEA2. Future work can consider the development of a smaller hydrocarbon facility electrical system model that can with high credibility represent the integrated model to reduce the iteration time.

\section{Acknowledgments}

The authors acknowledge the support of the Power System Operation Department/Saudi Aramco Oil Company, Brunel University and King Fahd University of Petroleum \& Minerals.

\section{References}

[1] "Saudi Arabia historical peak demand", http://ecra.gov.sa/peak_load.aspx\#.VOg-5I05BKA, accessed March 2016.

[2] "Saudi Electrical Company 2014 annual report", https://www.se.com.sa/en-us/Lists/AnnualReports/Attachments 114/AnnualReport2014En.pdf, accessed March 2016.

[3] Attia A. El-Fergany and Almoataz Y. Abdelaziz, "Efficient heuristic-based approach for multi-objective capacitor allocation in radial distribution networks," IET Generation, Transmission \& Distribution, 2014, vol. 8, Iss.1, pp. 70-80.

[4] Gian Luca Storti, Francesca Possemato, Maurizio Paschero, Antonello Rizzi, Fabio Massimo Frattale Mascioli, "Optimal Distribution Feeders configuration for active power losses minimization by genetic algorthims," IEEE Conference, 2013, pp. 407-412.

[5] C. M. Huang, S. J. Chen, Y. C. Huang and H. T. Yang, "Comparative study of evolutionary computation methods for active-reactive power dispatch," IET Generation, Transmission \& Distribution, 2012, vol. 6, Iss.7, pp. 636-645.

[6] Muhammad T. Al-Hajr and M. A. Abido, "Multiobjective optimal power flow using improved strength pareto evolutionary algorithm (SPEA2)," IEEE, 11th International Conference of Intelligent System Design and Application (ISDA), Cordoba, Spain, November 22-24, 2011, pp. 1-7.

[7] Juan M. Ramirez, Xiomara Gonzalez and Miguel Nedina, "Reactive power handling by a multi-objective formulation," IEEE, North American Power Symposium (NAPS), August 4-6, 2011, pp. 1-5.

[8] Benemar Alencar de Souza and Angelo Marcio Formiga de Almeida, "Multiobjective optimization and fuzzy logic applied to planning of the volt/var problem in distribution systems," IEEE Teans. Power systems, 2010, vol. 25, No.3, pp. 1274 1281.

[9] S. R. Spea, A. A. Abou El Ela and M. A. Abido, "Multiobjective differential evolution algorithm for environmentaleconomic power dispatch", IEEE International Energy Conference, Manama, 2010,pp. 841-846.

[10] M. Varadarajan and K. S. Swarup, "Solving multi-Objective optimal power flow using differential evolution", IET Generation, Transmission \& Distribution, 2008, vol. 2, No. 5, pp. 720-730. 